\title{
Placement of Wavelength Converters in Dynamically Routed All Optical Networks in presence of Tunable Transceivers
}

\author{
Shilpa S. Patil \\ Dept. of Electronics and Telecommunication, Maharashtra Institute of Technology, \\ Pune - 411038, India \\ E-mail: shilpapgaikwad@gmail.com
}

Bharat S. Chaudhari

Dept. of Electronics and Telecommunication, Maharashtra Institute of Technology, Pune - 411038, India

E-mail: bharat.chaudhari@mitpune.edu.in

Received: November 12, 2015 Accepted: December 22, 2015 Published: December 31, 2015 DOI: 10.5296/npa.v7i4.8567

URL: http://dx.doi.org/10.5296/npa.v7i4.8567

\begin{abstract}
Efficient routing with optimal resources is one of the challenging tasks in the design of DWDM networks. Wavelength Converter (WC) is an important resource, as the placement of WCs affects the network performance and the quantity of WCs affects the cost of the network. With the help of WCs the network performance can be maximized by removing the wavelength continuity constraint. As WCs are very expensive, selecting the candidate nodes for the placement of WCs in a network is important. In this paper we have proposed an optimized approach for the placement of WCs in the presence of tunable transceivers (TTRs) and fixed transceivers (FTRs). The performance analysis has been carried out for above approaches. Observation shows that sparse partial wavelength converters with various loads require only $2.4 \%$ converters. When tunable transceivers are used, an average reduction of $73 \%$ in blocking probability and average saving of $91 \%$ in required number of converters.
\end{abstract}

Keywords: Blocking Probability, FTR, RWA, TTR, Wavelength Converter. 


\section{Introduction}

In optical networks, wavelength division multiplexing (WDM) technology provides enormous bandwidth for long haul optical deployments. All optical circuits are used to establish the connections between end to end nodes without E-O and O-E conversions. To establish a lightpath between two nodes, same wavelength must be used on all the hops of the path. This constraint of continuous availability of the same wavelength is called wavelength continuity constraint (WCC). An efficient RWA algorithm [1] [2] is needed to improve the blocking performance of the network by the maximum utilization of the wavelengths. Further WCC diminution can be removed with the help of wavelength converter (WC) [3]. Wavelength convertible routers having wavelength conversion capability converts data on one wavelength into data on another wavelength. Different architectures have been proposed such as full wavelength conversion, share per link and share per node [4] for the placement of WCs. In full wavelength conversion, dedicated WCs are used at each port. Total converters required is the product of wavelengths per link and number of fibers used. In share per link, WC bank can be shared by the connection requests coming from each link whereas in share per node architecture, a common WC bank is present which is shared across links. In this if wavelength does not require conversion for establishment of lightpath then it is directly given to multiplexer for desired output. This entails very less number of WCs with high switching complexity and high sharing efficiency [4]. Each end node is equipped with few transceivers. Tunable transceiver can be tuned to different wavelengths so as to send out (or receive) an optical signal on any available free wavelength within its tuning range. This paper presents an optimized approach for the placement of WCs in the presence of tunable transceivers (TTRs) along with efficient routing scheme using dynamic link weight. Section II of the paper discusses the related work. Section III presents proposed work. Results, analysis and discussion are presented in Section IV whereas paper is concluded in Section V.

\section{Related work}

Network topology, definite number of converters and dynamic traffic is given, then selection of number of nodes for the placement of WCs becomes a very crucial task. There are different techniques proposed for the optimal placement of WCs in a network. Arora, Subramaniam [5] proposed Total Outgoing Traffic (TOT) for the placement of wavelength converters in the network. The nodes from which maximum routes passed are selected for the placement of WCs. In [6] authors have proposed a new technique called as weighted least congested routing (WLCR) with first-fit wavelength assignment in which current traffic load as well as route length is considered jointly. The routes are calculated off-line for every source and destination and the weight is assigned to each route. For every new connection request, the weight value is calculated and then assigned to each route. They have used Minimum Blocking Probability First (MBPF) heuristic technique in which each time wavelength converters are placed at each node and calculated the reduced blocking probability. Accordingly, the nodes are selected for the placement of the wavelength converters. Lot of research work has been done on the optimum placement of sparse partial 
WCs (SPWC) in which only few nodes are equipped with wavelength converters but with limited range capability. It is shown that small amount of wavelength converters can gain the performance which is nearly equal to the full WCs [7]. In such scenarios only certain numbers of wavelength routers are capable of doing the conversion [8]. Chu et al. [9] developed a method for the placement of WCs as SPWC architecture using fixed shortest path routing algorithm and first fit wavelength assignment. Initially by considering full WCs placement, value of total utilization of WCs at every node is calculated as average busy number of WCs and maximum busy converters. The nodes having maximum values can be replaced by placing the WCs. Static shortest path routing is used for finding out the routes. This architecture requires only $1 \%-5 \%$ of full WCs for varied traffic conditions. In [10], the auxiliary graph is created depending on the link state information of the route. The information is acquired from the history of number of converters utilized and idle converters from the intermediate nodes for the placement of wavelength converters. Least Loaded Routing (LLR) is used for the selection of the route. One or very few wavelength converters are placed on every node to reduce the blocking probability by $44 \%$ and $83 \%$. Fernando Lezama et al. [11] proposed Differential Evolution for the optimal placement of wavelength converters. The shortest path routing is used for finding out the routes. For assigning the wavelength random wavelength assignment technique is used. The algorithm considered population of individuals and use the iterations by building another populations till an optimal solution is achieved. Efficient and scalable wavelength assignment technique in [12] is used for the reduction of total number of WCs in wavelength routed networks. They have used a simple routing technique and for the wavelength assignment the wavelength which is free maximum number times is selected by graph coloring method. For ring network with unprotected and protected services, it is found that with the help of tunable transceivers further relaxation of the WCC and reduction in blocking probability can be achieved. In proposed work, we present a technique for finding out the positions of the nodes to place the WCs with dynamic weight based routing in the presence of tunable transceivers.

\section{Proposed work}

\subsection{System Assumptions and Notations}

NSFnet topology has been considered with $L$ links and $N$ nodes as shown in Fig. 1 for carrying out the simulation and analysis. The lightpath request is a Poisson process with arrival rate $\lambda$ and the holding times are exponentially distributed with average length $1 / \mu$. The traffic load behavior depends on Erlang model [13]. For every call request, the path is selected with the route having maximum dynamic weight value which can be calculated [14] on the basis of networks current situation of the network as in (7). From the total $k$ paths which are having free wavelengths for serving the lightpaths, the route with maximum dynamic weight is selected. The routes are calculated with the help of modified Dijkstra's algorithm. The wavelength is assigned with first fit wavelength assignment technique. Share per node architecture is considered for the placement of wavelength converter. The total blocking probability of the network is calculated by supervising the network for an hour. 


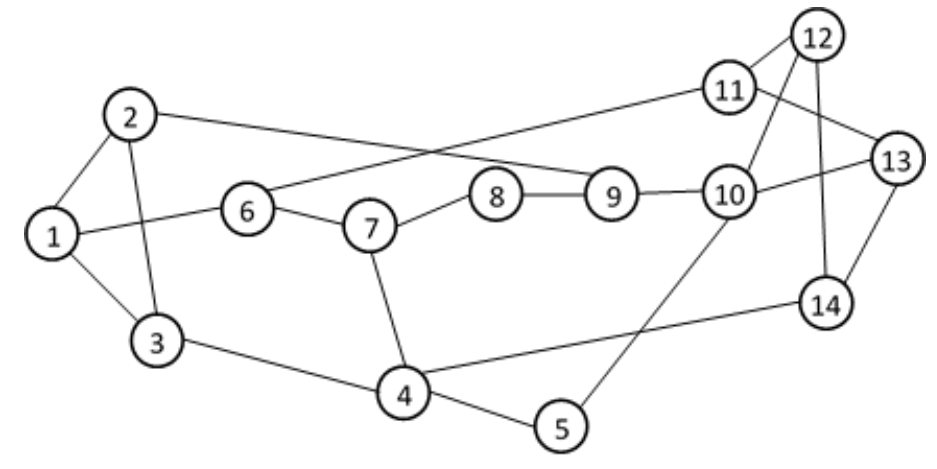

Fig. 1. NSFnet Topology

\subsection{Blocking Probability:}

The overall blocking probability of a network can be calculated as the ratio of blocked calls to the offered calls. The lighpath connection is blocked when wavelength is not available with dynamic RWA and unavailability of WCs in the presence of tunable transceivers. The overall blocking probability of the network can be calculated as

$$
\begin{gathered}
P_{N}^{b}=\frac{B_{N}^{C}}{A_{N}^{C}} \\
P_{N}^{b}=\frac{B_{1}^{C}}{A_{1}^{C}}+\frac{B_{2}^{C}}{A_{2}^{C}}+\frac{B_{3}^{C}}{A_{3}^{C}}+\cdots \cdots \cdots \cdots \cdots \cdots+\frac{B_{N}^{C}}{A_{N}^{C}}
\end{gathered}
$$

where $A_{N}^{C}$ is offered traffic on network and $B_{N}^{C}$ is total blocked calls by network for $N=\{n \mid n \in N, 1 \leq n \leq 14\}$.

\subsection{Candidate node selection for placement of WCs}

Optimal placement of WCs for dynamic traffic in a mesh topology is a NP- hard problem [15]. Finding the candidate nodes for the placement of WCs based on their blocking probabilities is a problem analogous to the clustering of a set of data points. Initially, blocking probabilities are calculated using dynamic weighted RWA algorithm and assuming that there are no wavelength converters in the network. These blocking probabilities are used as an input for the K-means clustering algorithm. K-means algorithm is used to minimize the objective function as given below,

$$
\arg \min \sum_{i=1}^{k} \sum_{X \in S_{i}}\left\|X-\mu_{i}\right\|^{2}
$$

where $\mu_{i}$ is the mean of points in $S_{i}$. Given a set of observations $X=\left\{X_{i} \mid 1 \leq i \leq n\right\}$ where each observation is a d-dimensional real vector, K-means clustering aims to partition the $n$ 
observations into $k(\leq n)$ sets $S=\left\{S_{i} \mid 1 \leq i \leq k\right\}$ so as to minimize the intracluster sum of squares.

\subsection{Algorithm to get candidate nodes for placement of WCs}

Inputs: Set of feature vector, $X=\left\{X_{i} \mid 1 \leq i \leq N\right\}$

where $X$ is set of blocking probabilities for all nodes calculated by assuming that there are no wavelength converters in the network and $X_{i}$ is the blocking probability of node $i$.

Number of clusters, $k=2$

Steps:

1. Randomly select $k$ cluster centers.

2. Calculate the distance between each data point and cluster centers using (4).

$$
d\left(X, \mu_{i}\right)=\sqrt{\left|X-\mu_{i}\right|^{2}}
$$

3. Assign the data point to the cluster center whose distance from the cluster center is minimum of all the cluster centers.

4. Recalculate the new cluster center using (5).

$$
\mu_{i}=\left(\frac{1}{k_{i}}\right) \sum_{j=1}^{k_{i}} X_{j}
$$

where $k_{i}$ represents the number of data points in $i^{\text {th }}$ cluster.

5. Recalculate the distance between each data point and new obtained cluster centers.

6. If no data point was reassigned then stop, otherwise repeat from step 3.

7. Select the cluster corresponding to the maximum blocking probability node.

8. Provide the WCs at all the nodes in the selected cluster.

Example:

Input data: Blocking probabilities of nodes $\left(B_{p}\right)$.

$B_{p}=\left\{\begin{array}{l}0.0005,0.0022,0.0011,0.0196,0.0005,0.0162,0.0094,0.0000,0.0122,0.0084,0.0084,0.0063, \\ 0.0008,0.0000,0.0021\end{array}\right\}$

Number of clusters: 2.

Result:

Cluster $1=\{0.0196,0.0162,0.0094,0.0122,0.0084\}$ 


\section{Macrothink}

Cluster $2=\{0.0005,0.0022,0.0011,0.0005,0.0000,0.0063,0.0008,0.0000,0.0021\}$

The corresponding nodes with respect to Cluster 1 are $\{4,6,7,9$, and 10$\}$ and Cluster 2 are $\{1,2,3,5,8,11,12,13$ and 14$\}$. Maximum blocking probability cluster is cluster1and corresponding nodes are $\{4,6,7,9$, and 10$\}$. Selected candidate nodes are as shown in Fig. 2.

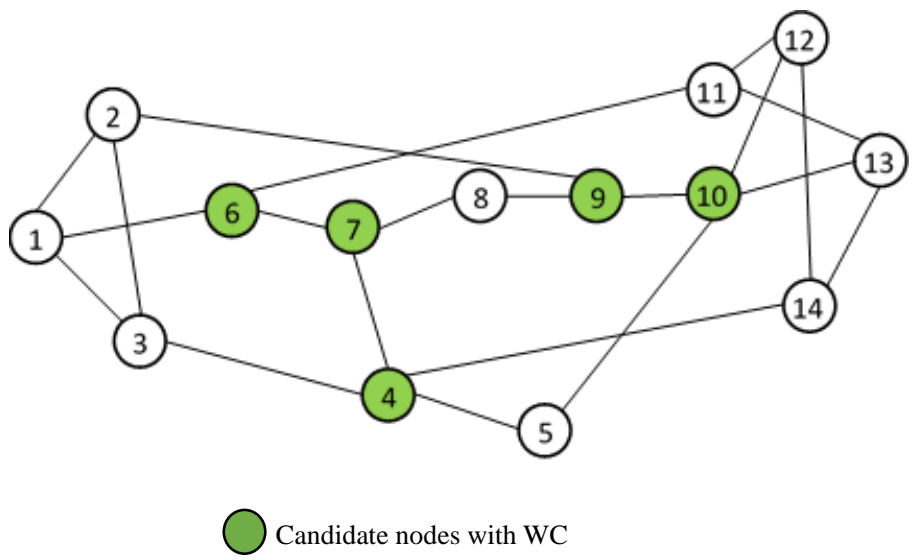

Fig. 2. NSFnet with selected nodes for the placement of WCs using proposed algorithm.

\subsection{Deciding the number of WCs at the candidate}

As the blocking probabilities varies from node to node, it is necessary to calculate the actual number of required converters at the particular node. Based on the blocking probability of a node, number of WCs can be calculated as [16] by using,

$$
C_{n}=C_{a} \cdot\left(\frac{\sum_{j=1}^{9} \sum_{i=1}^{14} \sum_{r=1}^{R_{n}} \frac{B_{c}^{i}}{B_{L 1}}}{S\left(N_{B}\right)} \times 100\right)
$$

where $C_{a}$ is number of available converters, $B_{c}^{i}$ is total blocked calls at node for $3600 \mathrm{~s}$,

$B_{L 1}$ blocked calls of network at load $L_{1}$ and $S\left(N_{B}\right)$ sum of average blocking calls for different loads for all 14 nodes. Depending on calls blocked and available pool of WCs, appropriate number of WCs are placed at selected candidate nodes.

\subsection{Optimal path selection based on link weight}

The efficient weight dependent dynamic Routing and Wavelength Assignment algorithm is used for the establishment of lightpath. The optimal weight value is calculated for each route as in [14] which depends on the networks current situation. The optimal path for efficient routing can be selected by using, 


$$
P_{W, \max }^{k}=\arg _{\max _{k=1} \text { to } 18}\left(\frac{\left(W_{a}\right)\left(T_{e}\right)\left(\sum_{i=1}^{P l} S_{c}(i)\right)}{\left[\left(\sum_{i=1}^{P l} S_{c}(i)\right)+\left(\sum_{i=1}^{P l} B_{c}(i)\right)\right]\left(\sum_{i=1}^{P l} h_{t}(i)\right)}\right)
$$

where $W_{a}$ is total number of available wavelengths on $k$ routes, $T_{e}$ is time elapsed, $S_{c}(i)$ is total number of served calls, $B_{c}(i)$ is total number of blocked calls, and $\mathrm{Pl}$ is path length of served calls.

\section{Experimental Analysis and Results}

Simulations have been carried out for the performance comparison of different converter placement schemes for NSFnet topology. Number of WCs at selected candidate nodes by using (6) is as shown in Table I.

Table. 1 Actual number of WCs placed at selected candidate nodes

\begin{tabular}{|c|l|c|c|c|c|c|}
\hline \multicolumn{2}{|c|}{ Nodes } & 4 & $\mathbf{6}$ & $\mathbf{7}$ & $\mathbf{9}$ & $\mathbf{1 0}$ \\
\hline \multirow{2}{*}{ Required Converters } & Load=200E with $C_{a}=40$ & 9 & 9 & 7 & 8 & 7 \\
\cline { 2 - 8 } & Load=1616E with $C_{a}=120$ & 27 & 27 & 22 & 24 & 20 \\
\hline
\end{tabular}

By using (7) the optimal path can be selected and results of the blocking probabilities with various loads are shown in Table 2.

Table.2 Comparison of blocking probabilities with $\mathrm{W}=40$

\begin{tabular}{|c|c|c|c|}
\hline Load in Erlang & without WC & with full WC & with partial WC \\
\hline 267 & 0.030216 & 0.015396 & 0.02117 \\
\hline 290 & 0.03255 & 0.02387 & 0.028391 \\
\hline 315 & 0.039297 & 0.027749 & 0.03223 \\
\hline 332 & 0.05573 & 0.040006 & 0.047785 \\
\hline 345 & 0.069758 & 0.051568 & 0.060163 \\
\hline 360 & 0.077288 & 0.053416 & 0.061944 \\
\hline
\end{tabular}

Fig. 3 shows variation in blocking probabilities at the candidate nodes for various load by using dynamic weight based RWA algorithm without WC. It shows that the blocking probability varies from node to node as well as for different load conditions. 


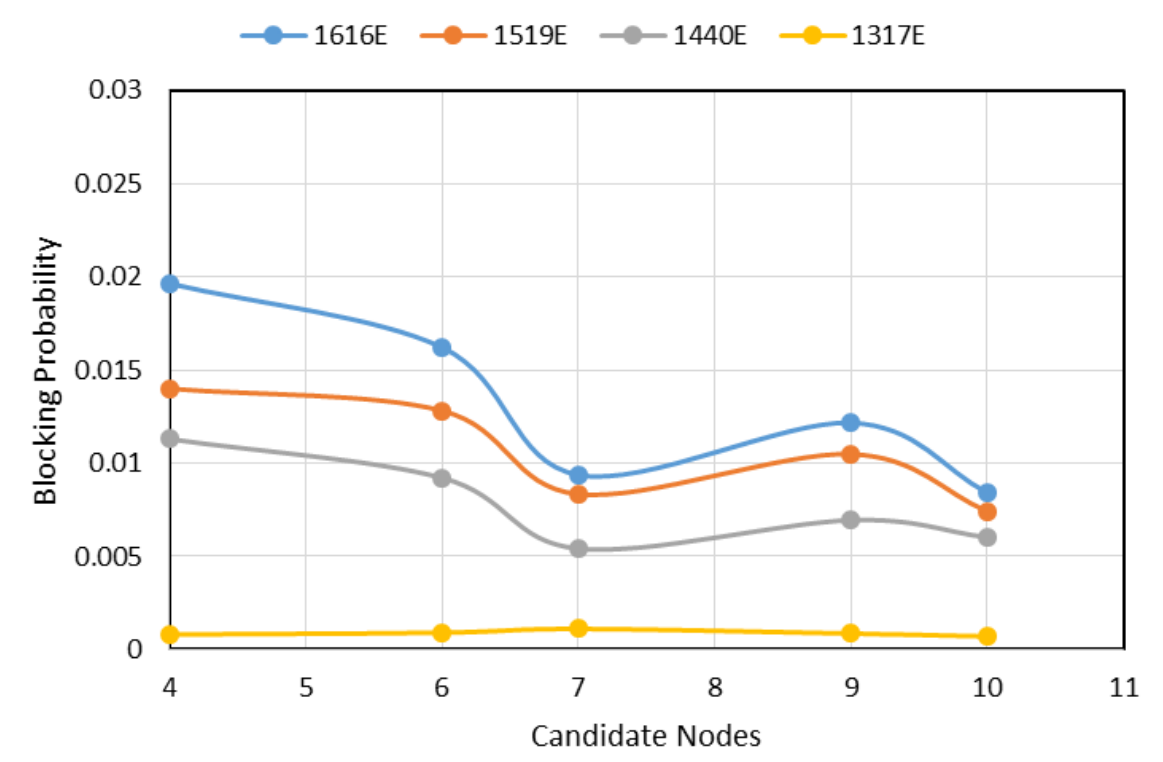

Fig. 3. Blocking Probability versus Candidate Nodes $(\mathrm{W}=120)$

WCs utilized versus load for various WC placement schemes with fixed transceivers (FTRs) and tunable transceivers (TTRs) for a given blocking probability shown in Fig. 4. The result shows that sparse partial WCs with TTRs require very less number of converters. Therefore it minimizes the complexity and cost of the network.

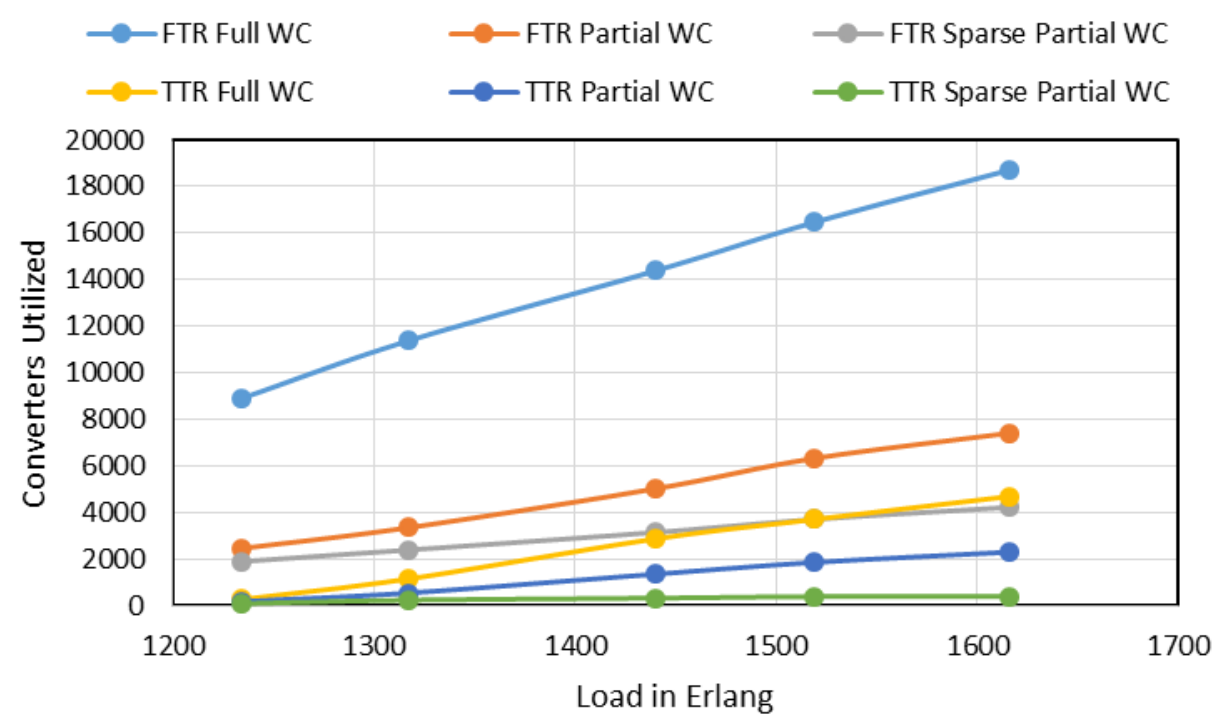

Fig. 4. Comparison of Converters Utilized versus Load $(\mathrm{W}=120)$

Fig. 5 shows the relationship between the blocking probabilities and load in Erlang for full WCs, partial WCs and sparse partial WCs with FTRs and TTRs. For partial WCs and sparse partial WCs the difference in blocking probability is very less and it is nearly equal to full WCs. It can be seen that the blocking probability can be reduced drastically with use of tunable transceivers along with appropriate number of WCs at properly selected nodes. 


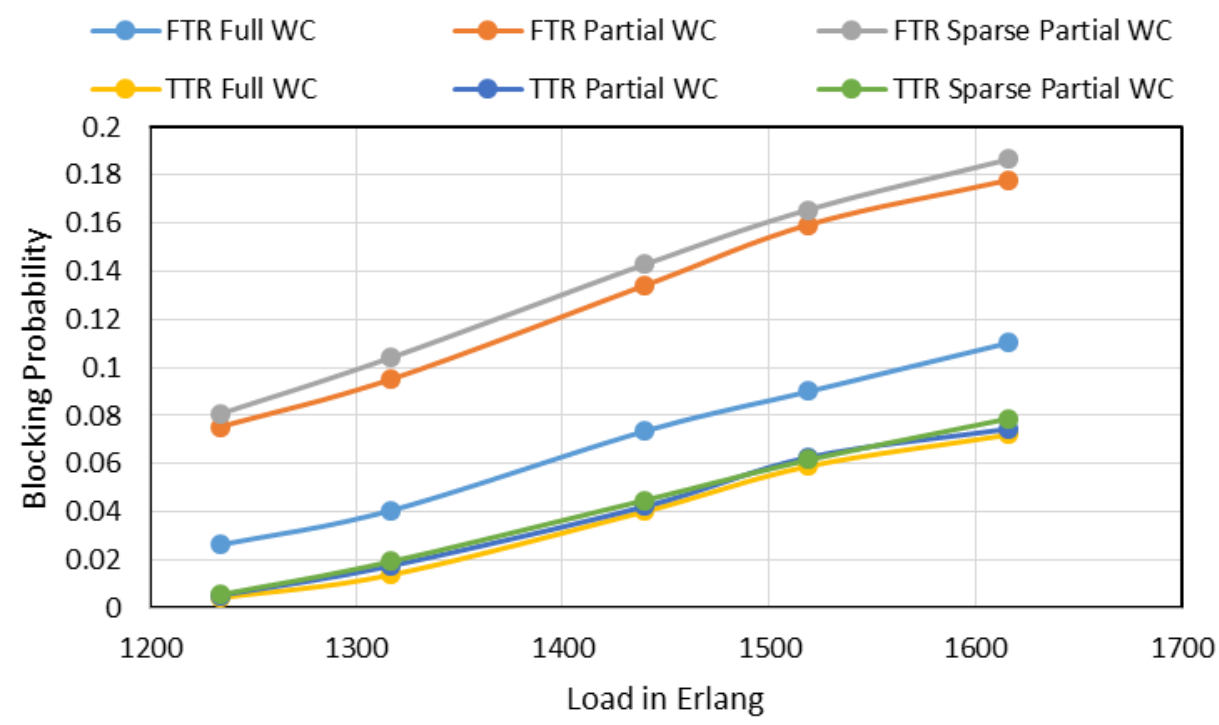

Fig. 5. Blocking Probability versus Load $(\mathrm{W}=120)$

Saving in percentage converters versus load for various WC placement techniques with TTR is as shown in Fig. 6. It shows that saving of average $84 \%$ converters with full WCs, average 88\% converters with partial WCs and average 91\% converters with sparse partial WCs when TTRs are used.

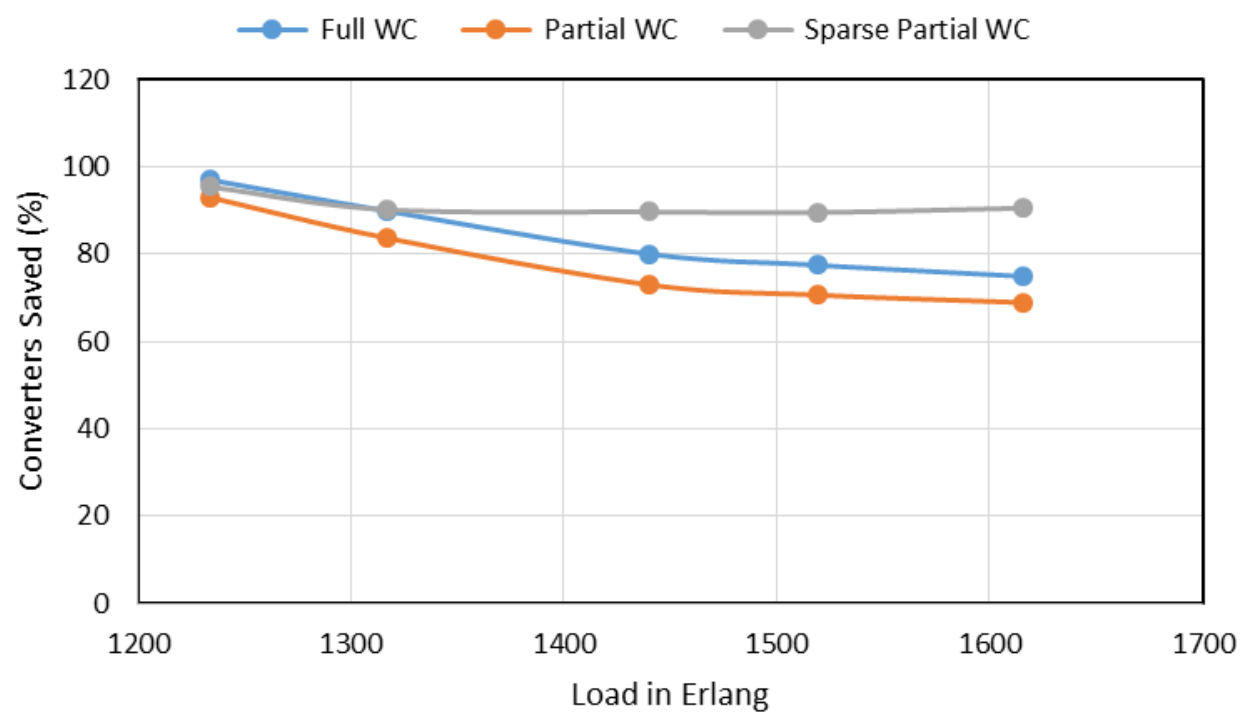

Fig. 6. Comparison of Converter Saved (\%) versus Load (W=120)

For partial WC five nodes are selected and only 40 WCs for low load and 120 WCs for high loads are used. On each link 40 wavelengths for low load and 120 for high load have been considered. With partial WCs approximately $12 \%$ wavelength converters are sufficient to get close performance to full wavelength converters. In sparse partial WC with variable number of WCs at the candidate nodes, only $2.4 \%$ WCs are sufficient to get the blocking performance near to the full WCs. 


\section{Conclusion}

Performance enrichment is obtained by using proper placement of WCs. For the optimal placement of WCs, sparse partial WCs are studied with efficient dynamic RWA in the presence of fixed and tunable transceivers. With the help of dynamic weight based RWA total number of WCs in WDM optical networks are reduced. Tunable transceivers releases the wavelength continuity constraint across the connection path and has a remarkable impact on the total number of WCs. The network with sparse partial WCs and tunable transceivers shows the better results for percentage saving in converters and percentage reduction in blocking probability.

\section{References}

[1] J.Zhou, X.Yuan, “A study of dynamic routing and wavelength assignment with imprecise network state information”, Proceedings. International Conference on Parallel Processing (ICPP) Workshops 2002, Vancouver, British Columbia, Canada. 21-21 August 2002. http://dx.doi.org/10.1109/ICPPW.2002.1039732

[2]L. Li, A. K. Somani, "Dynamic wavelength routing using congestion and neighborhood information”, IEEE/ACM Transaction on Networking, Vol. 7, Pp 779-786, Oct. 1999. http://dx.doi.org/10.1109/90.803390.

[3] M. Kovacevic and A. Acampora, "Benefits of Wavelength Translation in All-Optical Clear-Channel Networks,” IEEE Journal on Selected Areas in Communications, Vol. 14, No. 5, Pp 868-880, June 1996. http://dx.doi.org/10.1109/49.510910.

[4] C. Siva Ram Murthy, Mohan Gurusamy, "WDM Optical Networks: Concepts, Design, and Algorithms”, Prentice Hall, 2002.

[5] Arora, A. S. Subramaniam, S., "Converter Placement in Wavelength Routing Mesh Topologies”, IEEE ICC 2000, Pp http://dx.doi.org/10.1109/ICC.2000.853705.

[6] X. W. Chu, B. Li, and Z. Zhang, "Dynamic Routing and Wavelength Assignment in the Presence of Wavelength Conversion for All-Optical Networks”, IEEE/ACM Transactions on Networking, Vol. 13, Pp 704-715, June 2005. http://dx.doi.org/10.1109/TNET.2005.850226.

[7] Z. Zhang, C. M. Assi, A. A. Shami, M. A. Ali, and X. Liu, "Wavelength converters: improvement on the performance of optical networks," SPIE/Kluwer Optical Networks Magazine, Vol. 3, No. 2, October 2001. http://dx.doi.org/10.1117/12.445141.

[8] J S. Subramaniam, M. Azizoglu, and A. K. Somani, “All-Optical Networks with Sparse Wavelength Conversion,” IEEE/ACM Transactions on Networking, Vol. 4, No. 4, Pp 544-557, August 1996. http://dx.doi.org/10.1109/90.532864.

[9] Xiao Chu and Jiangchuan Liu, "Sparse partial wavelength conversion: Converter placement and wavelength assignment”. Optical Switching and Networking, Elsevier, Pp 66-74, 2010. http://dx.doi.org/10.1016/j.osn.2009.09.003.

[10]Yukinobu Fukushima, Takahiro Ooishi and Tokumi Yokohira, “A Wavelength and Converter Assignment Scheme Using Converter Usage History in Wavelength-Routed 
Networks”, International Journal of Computer Networks \& Communications, Vol. 5, No.4, Pp 63-73, July 2013. http://dx.doi.org/10.5121/ijcnc.2013.5406.

[11]Fernando Lezama, Gerardo Castanon, and Ana Sarmiento, "Differential evolution optimization applied to the wavelength converters placement problem in all optical networks”, Computer Networks, Elsevier, Pp 2262-2275, March 2012. http://dx.doi.org/10.1016/j.comnet.2012.03.019.

[12] M. Razo, S. Billenahalli, W. Huang, P. Monti, "Limiting wavelength converters usage in resilient wdm network", The IEEE Communications Society will hold its 2010 flagship conference (ICC 2010), Cape Town, South Africa. 23-27 May 2010. http://dx.doi.org/10.1109/ICC.2010.5501799.

[13] Hamzeh Berranvand and Jawad A. Salehi. "Multiservice Provisioning and Quality of Service Guarantee in WDM Optical Code Switched GMPLS Core Networks”, IEEE Journal of Lightwave Technology, Vol. 27, No. 12, Pp 1754-1762, 2009. http://dx.doi.org/10.1109/JLT.2009.2017499.

[14] S. S. Patil, B. S. Chaudhari and Baojun Li, "New Weight Dependent Routing and Wavelength Assignment Strategy for All Optical Networks in absence of Wavelength Converters”, ICTACT Journal on Communication Technology, Vol. 6, Issue 3, Pp 1131-1135, September 2015.

[15] R. S. Barpanda, A. K. Turuk, B. Sahoo, and Banshidhar Majhi, "Genetic Algorithm Approaches to Solve RWA Problem in WDM Optical Networks”, Swarm, Evolutionary, and Memetic Computing Conference, Chennai, Vol. 6466, Pp 599-606, December 2010. http://dx.doi.org/10.1007/978-3-642-17563-3_70.

[16] S. S. Patil and B. S. Chaudhari, "Placement of Sparse Partial Uniform and Non-uniform Wavelength Converters in Wavelength Routed Networks”, IEEE Connect Conference, Bangalore, July 2015.

\section{Copyright Disclaimer}

Copyright reserved by the author(s).

This article is an open-access article distributed under the terms and conditions of the Creative Commons Attribution license (http://creativecommons.org/licenses/by/3.0/). 